

\title{
Psychological Interventions for post-traumatic stress disorder in refugees and asylum seekers: A systematic review and meta-analysis
}

\author{
Dr Christopher T. Thompson ${ }^{1}$
}

Dr Andrew Vidgen ${ }^{2}$

\begin{abstract}
Dr Neil P. Roberts ${ }^{2,3}$
${ }^{1}$ South Wales Doctoral Programme in Clinical Psychology, Cardiff University.

${ }^{2}$ Psychology and Psychological Therapies Directorate, Cardiff and Vale University Health Board, Cardiff, UK
\end{abstract}

${ }^{3}$ Division of Psychological Medicine and Clinical Neurosciences, Cardiff University School of Medicine, Cardiff, UK

Corresponding author: Christopher Thompson.

Email: Christopher.thompson@wales.nhs.uk

South Wales Doctoral Programme in Clinical Psychology, 
School of Psychology

Cardiff University

Tower Building

70 Park Place

Cardiff

CF10 3AT

\section{Highlights}

- Increasing numbers of refugees and asylum seekers with complex needs.

- PTSD most-researched mental health condition for refugees.

- Available evidence to support use of trauma-focused psychological interventions.

- Lack of evidence for group-based or non-trauma-focused approaches at present.

- Evidence fairly consistent with the broader PTSD psychological intervention literature

\section{Keywords}

Refugees, asylum seekers, post-traumatic stress disorder, psychological intervention, metaanalysis 


\section{Abstract}

There is a high prevalence of post-traumatic stress disorder (PTSD) in refugee and asylum seeker populations which can pose distinct challenges for mental health professionals. This review included 16 randomised controlled trials (RCTs) with 1,111 participants investigating the effect of psychological interventions on PTSD in these populations. We searched PsychInfo, ProQuest (including selected databases ASSIA, IBSS, PILOTS), Web of Science, the Cochrane Central Database of Controlled Studies (CENTRAL) and Cochrane Database for Systematic Reviews (CDSR) to identify peer-reviewed, primary research articles up to June 2017. We used rigorous methods to assess the quality of included trials and evidence using Cochrane, SURE and GRADE systems. 525 trials were reviewed, 16 were included with 15 contributed to meta-analyses. Despite the challenges of conducting research in this field we found evidence for trauma-focused psychological interventions for PTSD in this population. Following sub-group analyses, we found evidence to support the use of EMDR and Narrative Exposure Therapy for PTSD symptoms. We considered these findings in relation to the broader PTSD treatment literature and related literature from survivors of large scale conflict. These findings suggest that trauma focused psychological therapies can be effective in improving symptoms for refugees and asylum seekers with PTSD.

\section{Introduction}

The number of forcibly displaced people around the world has increased by $75 \%$ over the last two decades with more individuals, families and communities affected by armed conflict, general violence and human rights violations (United Nations High Commission for Refugees UNHCR, 2016). The United Nations defines a refugee as someone who 'owing to a wellfounded fear of being persecuted for reasons of race, religion, nationality, membership of a 
particular social group, or political opinion, is outside the country of his nationality, and is unable to or, owing to such fear, is unwilling to avail himself of the protection of that country (UN General Assembly, 1951). An asylum seeker has asked a Government to provide them with refugee status and is awaiting a decision. Globally, in 2016 the UNHCR reported over 21 million refugees and close to 3.2 million asylum seekers.

Refugees and asylum seekers are much more likely to have experienced traumatic events than members of the general population in high income countries (Kalt, Hossain, Kiss, \& Zimmerman, 2013). Individuals who have experienced multiple traumatic events in their home country, in transition to, and within the hosting country, undergo elevated levels of stress linked with unmet basic needs and uncertainty about their own future and the safety of loved ones. These experiences cause huge personal losses which are a major threat to identity (Carlsson, Sonne, \& Silove, 2014). Refugees and asylum seekers have higher rates of mental health conditions, particularly post-traumatic stress disorder (PTSD), anxiety, depression (Bogic et al., 2015; Burnett \& Peel, 2001; Steel, Chey, Silove, Marnane, Bryant \& van Ommeren, 2009) and psychoses (Hollander et al., 2016). Of these mental health conditions, PTSD is the most widely researched in refugees and asylum seekers populations. PTSD is a major global health problem for refugees and asylum seekers worldwide. Fazel, Wheeler and Danesh (2005) estimated that refugees are ten times more likely to experience PTSD than the general population in a systematic review of the prevalence of serious mental health disorders for individuals living in high income countries.

Psychological therapies have been used in the treatment of PTSD in the general population since PTSD was first included in the Diagnostic and Statistical Manual of Mental Disorders (DSM-III) (American Psychiatric Association, 1980). Efficacious psychological interventions for the treatment of PTSD include trauma-focused cognitive behavioural therapy (TF-CBT) and eye-movement desensitisation and reprocessing (EMDR) (Bisson, Roberts, Andrew, Cooper, \& Lewis, 2013). The terms TF-CBT refers to a variety of cognitive behavioural 
therapy (CBT) including Prolonged Exposure (Foa, Hembree, \& Rothbaum, 2007), Cognitive Processing Therapy (Resick \& Schnicke, 1993), Narrative Exposure Therapy (NET) (Schauer, Elbert, \& Neuner, 2011) and Cognitive Therapy (Ehlers \& Clark, 2000). These therapies aim to support individuals to manage difficulties following traumatic events by combining cognitive therapy and behavioural therapy to change key maintaining factors in PTSD, e.g., exposing the individual to the distressing memory. NET has been adapted from established TF-CBT approaches specifically for multiply traumatised populations, such as refugees and survivors of war trauma (Schauer et al., 2011). Like other forms of TF-CBT NET involves exposure to traumatic memories but involves the reorganisation of these memories into a coherent chronological narrative. EMDR aims to support individuals to reprocess their traumatic memories and involves supporting the individual to focus on distressing components of the memories including the image, thoughts, feelings and physical sensations, whilst guiding them through sets of structured eye-movements in a process of bilateral stimulation. In recently adopted guidelines the American Psychological Association Guideline Development Panel (GDP; American Psychological Association, in press) has given strong recommendation for the use of Prolonged Exposure, Cognitive Processing Therapy and Cognitive Therapy and Cognitive Behavioural Therapy with additional weaker recommendations for EMDR and Narrative Exposure Therapy based on a lower strength of evidence. The APA guidelines do not make recommendations in relation to specific population. Refugees often face significant barriers to accessing appropriate mental health care. This is partly as a result of language and cultural barriers (Priebe, Giacco, ElNagib, 2016) but also as a result of legal/ political restrictions and attitudes of local services, which often results in them being unable to access appropriate evidence-based care available to indigenous groups (Langlois, Haines, Tomson \& Ghaffar, 2016). It is therefore important to establish whether refugees can potentially benefit from established interventions in order to advocate for appropriate care. 
Within the refugee field there has been a significant debate about the challenge of treatment related research with refugees (see Carlsson et al., 2014). These concerns include the possibility that refugees are too vulnerable to engage in research; ethical concerns about the appropriateness of randomisation for refugees and fears about the potential consequences of negative findings of future program funding. Other concerns include questions about the appropriateness of applications of Western trauma treatment models to refugees (see Nickerson, Bryant, Silove \& Steel, 2011). Some have argued that such models are potentially simplistic, medicalise the distress of refugees, ignore the importance of a human rights focus and potentially ignore cultural, social, historical and political meanings (see Nickerson et al., 2011; Patel, Williams, \& Kellezi, 2016; Steel et al., 2009). Some authors have been critical of the idea that therapeutic approaches based on treatment of PTSD in single trauma events have any validity when directly applied to refugees, arguing that such approaches ignore the complexity of need (Jaranson, Jacobs, Kinzie, \& Quiroga, 2006). Others have argued for the importance of PTSD based therapies, when indicated once primary needs for safety and security have been established (Turner \& Herlihy, 2009). Whilst giving recognition to these concerns about Western based treatment approaches some authors (Carlsson et al., 2014; Nickerson et al., 2011) have argued that pragmatic intervention evaluation is essential to the dissemination and provision of effective intervention for traumatised refugees and a failure to collect evidence risks perpetuating forms of intervention, which may not be helpful. Nickerson and colleagues also make the case that it may sometimes be necessary for refugees to address very disabling traumatic stress symptoms before they can address other psychosocial problems.

Despite the concerns and challenges described, a number of trials of interventions for refugees with PTSD have been undertaken over the past 15 years. This work has led to a number of previous reviews. In an initial qualitative review of 10 randomised controlled trials (RCTs) (Crumlish \& O'Rourke, 2010) found evidence for CBT and NET, describing the latter as probably the best-supported modality. The authors concluded that no treatment for PTSD 
in refugees and asylum seekers had a solid evidence base. Another qualitative review (Robjant \& Fazel, 2010) of NET involving studies with refugees and asylum seekers summarised that studies had demonstrated efficacy in treating PTSD in a variety of low- and middle-income settings and for treating PTSD in refugees and asylum seekers in highincome settings. Nickerson et al (2011) undertook a systematic review of the evidence for trauma focused interventions for PTSD versus multimodal interventions aiming to address psychological, social, health related and cultural adaptation issues. They identified a number of studies reporting positive outcomes for trauma focused approaches and a small number of studies evaluating multimodal interventions. These studies of multimodal therapies did not find significant improvements in symptoms despite offering lengthy interventions. A metaanalysis (Lambert \& Alhassoon, 2015) of 12 RCTs examined the results of psychotherapeutic intervention for traumatised adult refugees. Comparisons of 13 traumafocused therapies found evidence for the benefit of psychological interventions for PTSD.

Refugees and asylum seekers are often victims of torture (Burnett \& Peel, 2001). A Cochrane review (Patel, Kellezi, \& Williams, 2014) compared nine RCTs of interventions for psychological health and well-being of torture survivors, a population with similarities to refugees and asylum seekers. The authors reported no immediate benefits of psychological therapy in comparison with controls for PTSD symptoms and PTSD caseness. However, they found evidence to support a moderate effect of CBT and NET at six months posttreatment. Evidence was described as being of very low quality with authors citing limitations as non-standardised assessment methods using interpreters and very small sample sizes. A recent review and meta-analysis (Nosè et al., 2017) of 14 controlled and uncontrolled trials compared psychosocial interventions with waitlist or treatment as usual in adult refugees and asylum seekers in high-income countries. The authors found significant benefits of psychological therapies in reducing PTSD symptoms and concluded that their findings provide empirical evidence that psychosocial interventions that are effective for PTSD in the general population may not completely overlap with those that are appropriate for PTSD in 
refugees and asylum seekers. Nosè and colleagues emphasise the limitations of evidence in their review citing a small number of studies and low methodological quality.

There are a number of limitations to previous reviews in terms of evaluation of intervention aimed at treating PTSD. Whilst all of the cited reviews have focused on trauma-exposed populations requirements in terms of PTSD has not normally been explicit. In addition, some have based their findings purely on a narrative synthesis (e.g. Crumlish \& O'Rourke, 2010; Robjant \& Fazel, 2010; Nickerson et al., 2011) and most have included both controlled and non-controlled studies. A further limitation of some reviews is that whilst they undertook evaluation of the methodologies of included studies, these evaluations were not systematic or structured and did not consider some of the key domains associated with risk of bias (Higgins \& Green, 2011). The review undertaken by Nosè and colleagues was published during the period when we were conducting our review and whilst we were aware of findings from this review we undertook to complete our review independently. Since publication of this and other reviews a number of new randomised controlled trials (RCTs) have been completed, including several trials evaluating EMDR (Acarturk et al., 2015, 2016; ter Heide, Mooren, van de Schoot, De Jongh, \& Kleber, 2016) not included in previous reviews (Lambert \& Alhassoon, 2015; Nosè et al., 2017). Previously, no meta-analysis has assessed the efficacy of EMDR when considering the effect of psychological interventions. We aimed to build on the review of Robjant \& Fazel (2010) by including a number of new trials (HenselDittmann et al., 2011; Hijazi et al., 2014; Stenmark, Catani, Neuner, Elbert, \& Holen, 2013) investigating NET which were published subsequently. We, therefore, took the view that the time was right to undertake a further systematic review, with meta-analysis where appropriate. We also believed that it was important to address some of the methodological limitations of these previous reviews; in particular to undertake a structured evaluation of the methodologies of included trials and to base our findings on RCTs only. We set limits on the proportion of participants who were not either an asylum seeker or refugee and made it necessary for a minimum of $80 \%$ of participants to have a probable PTSD diagnosis based 
on validated standardised assessment measures. We considered the appropriateness of including other highly traumatised populations such as those specifically affected by war and torture in this review. We recognise that such populations share common experiences with many refugees. However, we also recognised that there are some features and challenges which provide particular and additional challenges for refugees, by virtue of the degree of loss and displacement they typically experience and the particular challenges of adapting to new cultures and societies (Carlsson et al., 2014). Finally, we included studies from low-, middle- and high- income settings. We set out to build on previous work by undertaking a review based on the guidelines set out by the Cochrane Collaboration (Higgins \& Green, 2011). Our objective was to determine the efficacy of psychological interventions aimed at treating PTSD in adults who are seeking asylum or have refugee status. This review aimed to present an up-to-date analysis of randomised controlled trials (RCTs). We anticipated that we would only be able to identify a small pool of relevant studies and that this would limit the statistical power of potential findings. We therefore set out to interpret findings in terms of the broader PTSD treatment evidence base and in light of the particular challenges we have described in conducting research trials with this population.

\section{Methods}

\section{Criteria for considering studies for this review}

Randomised, controlled trials with a primary focus to investigate the clinical efficacy of psychological interventions for treating PTSD in adults were considered for inclusion in this review. The RCT is generally considered to be the gold standard in treatment outcome research (Ehring et al., 2014), providing a reduced risk of bias and the most robust means of furthering evidence of the effectiveness of clinical interventions (Higgins \& Green, 2011). We specified that studies must include a control condition, (e.g. treatment as usual, waitlist 
control, monitoring group) or an alternative psychological intervention condition and must be a primary research paper. Included psychological interventions were those which had been reviewed by the Cochrane review of psychological therapies for PTSD in adults (Bisson et al., 2013). These included individual and group therapies, with or without trauma-focused techniques. Studies were limited to peer-reviewed, English-language papers only. Study sample size was not used to limit selection. This review defined adults as 18 years or over and required $80 \%$ of study participants to be either an asylum seeker or a refugee at point of recruitment. In line with prior PTSD treatment outcome reviews (Roberts, Roberts, Jones, \& Bisson, 2015) $80 \%$ of study participants were required to have a probable PTSD diagnosis at point of recruitment according to DSM-III (APA 1980), DSM-IIIR (APA 1987), DSMIV (APA 2000), DSMV (APA 2013), ICD-9 (WHO 1979) or ICD-10 (WHO 1992) criteria. Accepted methods of PTSD diagnosis or probable diagnosis required either a clinician-led structured interview (e.g. the Clinician Administered PTSD Symptom Scale CAPS, (Blake et al., 1995) or a self-report measure validated for PTSD diagnosis (e.g. the Posttraumatic Diagnostic Scale PDS, (Foa, Cashman, Jaycox, \& Perry, 1997). There was no restriction on type of traumatic event, other co-morbidity, substance use or study setting.

To identify studies, systematic computerised searches of Psychlnfo, ProQuest (including selected databases ASSIA, IBSS, PILOTS), Web of Science and the Cochrane Central Database of Controlled Studies (CENTRAL) from 1 January 1992 to 11 June 2017 were carried out. The following search terms and Boolean operators were used $\mathrm{Tl}=($ asylum seeker* OR refugee*) AND TI=(intervention* OR treatment* OR therap* OR RCT OR "randomised control* trial" OR "randomized control ${ }^{*}$ trial") NOT TI=(child* OR adolescent* OR school). Additionally, the Cochrane Database for Systematic Reviews (CDSR) was searched for relevant reviews. We checked reference lists of reviews identified and those of included studies. 


\section{Outcomes}

The primary outcome identified was PTSD symptom severity using standardised and validated assessment measures. The three secondary outcomes were: depressive symptom severity, PTSD diagnostic status and participant drop-out as measured by the number of participants who had retained in treatment. For PTSD severity primacy was given to standardised clinician-administered assessments. Following from Bisson et al., (2015), outcome time points were grouped into four month periods of 0 to 4 months for postintervention, and between 5 to 8 months, 9 to 12 months and 13 months or more for follow up.

\section{Data extraction}

The titles and abstracts of all potential trials were read. If an abstract appeared to represent a randomised, controlled trial with a primary focus to investigate the clinical efficacy of a psychological intervention for PTSD, two reviewers independently conducted a whole article review to determine if the study met the inclusion criteria. The Specialist Unit for Review Evidence (SURE) checklist for experimental studies (Specialist Unit for Review Evidence (SURE), 2013) was used to capture study characteristics and study data. Any differences regarding study inclusion were discussed and presented to a third reviewer when agreement could not be obtained.

\section{Assessment of methodological quality}

We used the Cochrane Collaboration's handbook for assessing risk of bias which provides an established framework for evaluating the quality of evidence of findings from systematic reviews (Higgins \& Green, 2011). The approach focuses assessment on seven key areas of methodological quality: sequence generation, allocation sequence concealment, blinding of participants and investigators, blinding of outcome assessment, incomplete outcome data, selective outcome reporting and other biases. As the blinding of participants and 
investigators is extremely difficult to achieve within psychological studies, this criterion was excluded. We used the SURE checklist for experimental studies (Specialist Unit for Review Evidence (SURE), 2013) to support the quality assessment process. The checklist comprises 14 broad categories with specific questions regarding internal validity, such as clearly defined hypotheses, methodology and validity of results. The checklist also supports analysis of key quality areas not included within the Cochrane approach, such as sample size, power analysis and the granting of ethical approval. We decided to provide a risk of bias table including the seven Cochrane risk of bias domains, as well as additional domains covered by the checklist, to improve transparency and reliability of the review process. The risk of bias for each criterion was rated as of high, low or unclear risk of bias in accordance with Cochrane guidelines (Higgins \& Green, 2011). We contacted authors to try to obtain clarification when there was insufficient information to make a risk of bias judgement.

\section{Main and subgroup analyses}

We conducted an initial analysis of any psychological intervention versus waitlist or treatment as usual. We made an a priori decision to analyse group based interventions separately from individual interventions. We also decided to undertake subgroup analyses according to the type of psychological intervention i) EMDR ii) NET iii) CBT, and type of control condition i) inactive (e.g. waitlist/treatment as usual) ii) active (e.g. alternative psychological intervention). The time point for the primary analyses was post intervention (04 months) with follow-up time points investigated for main outcomes as a subgroup analysis.

\section{Statistical analyses and quality of evidence}

We used the standardised mean difference (SMD) to analyse continuous outcomes as trials measured outcomes on different scales. SMD was based on Hedges' g (Hedges, 1981) calculated by dividing the difference in mean outcome between groups by the standard deviation of outcome among participants. We used risk ratio $(\mathrm{RR})$ to measure categorical 
outcomes. Heterogeneity between studies was examined by observing the $\mathrm{Q}$ statistic $(p<$ $0.10)$ and the $\mathrm{I}^{2}$ statistic. A random-effects model was used to summarise all results as we anticipated a large degree of clinical and methodological diversity between studies. Review Manager Version 5.3.5 (The Cochrane Collaboration, 2011) was used to analyse data. All $p$ values are 2-tailed. We assessed the quality of evidence using the "Grades of Recommendation, Assessment, Development, and Evaluation" (GRADE) approach (Guyatt et al., 2013; Guyatt, Oxman, Schünemann, Tugwell, \& Knottnerus, 2011) which provides an established framework for evaluating the quality of evidence of findings from systematic reviews (Higgins \& Green, 2011). The quality of evidence was assessed using five factors: limitations in study design and implementations of included studies, unexplained heterogeneity or inconsistency of results, potential publication bias, imprecision of effect estimates. We pooled data to provide an overview classification of the quality of evidence according to the following criteria:

- High quality: further research is very unlikely to change our confidence in the estimate of effect.

- Moderate quality: further research is likely to have an important impact on our confidence in the estimate of effect and may change the estimate.

- Low quality: further research is very likely to have an important impact on our confidence in the estimate of effect and is likely to change the estimate.

- Very low quality: we are very uncertain about the estimate

From a starting point of high quality, the rating of quality was downgraded by one level for each serious study limitation (risk of bias), or two levels for very serious limitations, relating to indirectness of evidence, serious inconsistency, imprecision of effect estimates or potential publication bias. 


\section{Results}

\section{Insert Figure 1 about here.}

A final search was conducted on 29 May 2018 and identified 525 unique citations 384 were removed after reading the title and abstract. A further 127 studies were excluded following a full paper review. The reasons for exclusion are provided in Appendix A. Sixteen studies with 1,111 participants met inclusion criteria and were included with fifteen of these providing data which contributed to meta-analysis. The mean study sample size was 69 participants (range 10 to 280). The mean length of follow-up was five months (range 1 to 12 ). Figure 1. provides a PRISMA flow chart of the selection criteria.

\section{Study characteristics}

Study characteristics of included studies are provided in Table 1. One study involved asylum seekers only (Neuner, Kurreck, Ruf, Odenwald, \& Schauer, 2010), six studies involved refugees only (Buhmann, Nordentoft, Ekstroem, Carlsson, \& Mortensen, 2016; Hijazi et al., 2014; Hinton et al., 2004, 2005; Otto et al., 2003; Paunovic \& Ost, 2001), five studies involved both refugees and asylum seekers and analysed their outcomes either together (Adenauer et al., 2011; Hensel-Dittmann et al., 2011; Ter Heide, Mooren, Kleijn, de Jongh, \& Kleber, 2011; Ter Heide et al., 2016) or separately (Stenmark et al., 2013) and four studies involved forcibly displaced migrants currently residing in refugee camps, with two studies in Turkey and two in Uganda (Acarturk et al., 2015, 2016; Neuner et al., 2008; Neuner, Schauer, Klaschik, Karunakara, \& Elbert, 2004). Eight studies were conducted in Europe (Norway, Sweden, Denmark, three in Germany, two in The Netherlands) and four in the USA. Interpreters were used in ten of the studies.

Insert Table 1. about here 


\section{Interventions and control conditions}

All main psychological interventions investigated by included trials were identified as traumafocused as they involved a substantive exposure component.

\section{Individual trauma-focused approaches}

Five studies (Adenauer et al., 2011; Hijazi et al., 2014; Neuner et al., 2008, 2010; Stenmark et al., 2013) compared Narrative Exposure Therapy (NET) with an inactive control. The number of treatment sessions ranged from 3 to 12 with a mean of 7 sessions. Three studies (Hensel-Dittmann et al., 2011; Neuner et al., 2008 2004) compared NET with an active control. Hensel-Dittmann et al. (2011) compared 10 sessions of NET with 10 sessions of stress inoculation therapy. Neuner et al. (2008) compared six sessions of NET with six sessions of trauma counselling and aimed to investigate whether trained counsellors recruited from a population of refugees, could carry out effective treatment for PTSD in a refugee camp. Stenmark et al. (2013) compared 10 sessions of NET delivered by a range of healthcare professionals including nurses, occupational therapists, and social workers, with a treatment as usual control condition.

Four studies (Acarturk et al., 2015, 2016; ter Heide et al., 2011, 2016) investigated Eye Movement Desensitisation and Reprocessing (EMDR) with two studies (Acarturk et al., $2015,2016)$ comparing seven sessions of EMDR with a waitlist control. Two studies (ter Heide et al., 2011, 2016) compared EMDR with stabilisation with ter Heide et al. (2016) comparing 9 sessions of EMDR with 12 sessions of stabilisation and ter Heide et al. (2011) comparing 11 EMDR sessions with 11 stabilisation sessions. Participants were divided evenly between groups in all studies. Studies by Acarturk and colleagues were conducted in a refugee camp with culturally sensitive treatment carried out in a kindergarten to avoid stigma associated with mental illness. 
Four studies investigated individual Cognitive Behavioural Therapy (CBT) adapted for trauma (Buhmann et al., 2016; Hinton et al., 2004, 2005; Paunovic \& Ost, 2001). Three studies (Buhmann et al., 2016; Hinton et al., 2004, 2005) compared CBT with an inactive control. The number of treatment sessions ranged from 12 to 16 with a mean of 13.3 sessions. Paunovic \& Ost (2001) compared 16 to 20 sessions of CBT with 16 to 20 sessions of exposure therapy. The study by Buhmann and colleague was based on a $2 \times 2$ design evaluating CBT and Sertraline in combination and separately.

\section{Group-based approaches}

We only found one study of a group based intervention. This study involved psychological treatment in a group format compared with an active control. Five participants underwent 10 sessions of trauma-focused CBT for adults in a treatment group whilst taking sertraline compared to a control condition with five participants undertaking a course of sertraline only.

\section{Insert Table 2 about here.}

\section{Quality of methodology}

The methodological quality of studies is shown in Table 2. A detailed table on Cochrane risk of bias criteria is provided in Appendix B. The majority of studies used appropriate methods for sequence generation, allocation sequence concealment and blinding of outcome assessment and were judged as low risk. Eleven studies provided complete outcome data with five studies omitting outcome data. It was largely unclear as to whether studies selectively reported outcomes although four studies did detail their outcomes in a trial protocol. Other biases included potential researcher allegiance to intervention with six studies evaluating NET including researchers who have co-authored intervention protocols (Adenauer et al., 2011; Hensel-Dittmann et al., 2011; Neuner et al., 2008, 2010, 2004; 
Stenmark et al., 2013). Several therapist effects were reported in four trials (Hijazi et al., 2014; Hinton et al., 2004, 2005; Paunovic \& Ost, 2001) with either one or two therapists carrying out all treatment, and in one trial where one therapist was believed not to agree with the treatment (ter Heide et al., 2011). In a small number of studies, authors reported that participants could have an incentive to underreport progress if believing doing so could benefit their asylum claim. Therapist and assessor training, level of experience and professional backgrounds varied considerably between studies. Ten studies reported using interpreters.

Thirteen studies included a clearly defined hypothesis. Two studies did not clearly define their interventions or did not clearly distinguish treatment components between interventions. Nine studies reported obtaining ethical approval. Whilst it was assumed that most studies would have obtained ethical approval, two studies did not report doing so and one did not confirm approval being granted. Only five studies registered a trial protocol prior to trial commencement. Six studies were rated at high or unclear risk of bias from groups potentially being dissimilar at the start of the trial due to factors including baseline characteristics, degree of or types of trauma, length of time living in country. The sample size was small in nine studies (Acarturk et al., 2015; Adenauer et al., 2011; Hensel-Dittmann et al., 2011; Hinton et al., 2004; Neuner et al., 2010, 2004; Otto et al., 2003; Paunovic \& Ost, 2001; Ter Heide et al., 2011) and only six studies undertook a power analysis. Participants were properly accounted for in two trials with unclear accounting in 11 trials and high risk in three trials. An intention-to-treat analysis was carried out in nine trials with no need in two trials due to all participants remaining until follow-up. Four trials did not attempt an intention-totreat analysis and one trial carried out an insufficient analysis. Ten studies reported sufficient data analysis including statistical and analytical methods, providing estimates of effect size, and meaningful confident intervals. Information provided was unclear in six studies. Results provided were seemingly reliable based on outcome measures used, outcomes assessed and authors' conclusions being adequately supported by the results. This was unclear in 
eight studies. Seven studies did not report on sponsorship or potential conflicts of interest. Fourteen studies adequately identified limitations.

\title{
Insert Table 3 about here.
}

\section{Assessed outcomes and evidence synthesis}

Outcomes were grouped and analysed in line with intervention characteristics identified above and decided a priori as recommended by Cochrane guidelines (Higgins \& Green, 2011). The results of meta-analyses for the main outcome PTSD severity are shown in Table 3.

\author{
Main Analysis \\ Individual trauma-focused psychotherapy (TFP) vs waitlist control/treatment as \\ usual or minimal intervention
}

Ten trials included in this review evaluated an individual psychological intervention versus waitlist control/treatment as usual or minimal intervention (Acarturk et al., 2015, 2016; Adenauer et al., 2011; Buhmann et al., 2016; Hijazi et al., 2014; Hinton et al., 2004, 2005, Neuner et al., 2008, 2010; Stenmark et al., 2013). Eight trials (Acarturk et al., 2015, 2016; Adenauer et al., 2011; Buhmann et al., 2016; Hijazi et al., 2014; Hinton et al., 2004, 2005; Stenmark et al., 2013) with 474 participants were involved in an analysis of individual TFP on PTSD severity at post-intervention. We found a large effect in favour of individual psychological intervention at post-intervention for PTSD severity (SMD $-1.14 ; 95 \% \mathrm{Cl}-1.80$ to -0.47 ) as shown in Figure 2. Two trials, both investigating NET, conducted 5-8 month follow-up analysis as described in the NET section below. We found a moderate effect in favour of NET (SMD $-0.62 ; 95 \% \mathrm{Cl}-0.93$ to -0.32$)$. 


\section{Insert Figures 2 about here.}

For secondary outcomes, seven studies reported data on depressive symptoms (Acarturk et al., 2015, 2016; Adenauer et al., 2011; Buhmann et al., 2016; Hijazi et al., 2014; Hinton et al., 2004; Stenmark et al., 2013) at post-intervention. We found a moderate effect (SMD $0.71 ; 95 \% \mathrm{Cl}-1.17$ to -0.25$)$ in favour of psychological interventions. Data were available from five studies for PTSD diagnosis (Acarturk et al., 2015, 2016; Adenauer et al., 2011; Stenmark et al., 2013; Ter Heide et al., 2016) with a difference in favour of psychological interventions (RR $0.63 ; 95 \% \mathrm{Cl} 0.41$ to 0.97 ). We found no difference between groups for participant drop-out (RR $0.81 ; 95 \% \mathrm{Cl} 0.65$ to 1.02$)$ based on data from eight studies (Acarturk et al., 2015, 2016; Buhmann et al., 2016; Hijazi et al., 2014; Hinton et al., 2005; Neuner et al., 2008, 2010; Stenmark et al., 2013). Using GRADE, we rated the quality of evidence for all findings as low to very low.

Individual trauma-focused psychotherapy (TFP) vs active control

Seven trials (Buhmann et al., 2016; Hensel-Dittmann et al., 2011; Neuner et al., 2008, 2004; Paunovic \& Ost, 2001; Ter Heide et al., 2011, 2016) with 517 participants contributed to an analysis of individual TFP compared with an active control. The Neuner et al. (2004) trial had three treatment arms: comparing NET with psychoeducation, and supportive counselling. We used data from the NET and supportive counselling arms in this comparison. No difference was found for PTSD severity (SMD $-0.03 ; 95 \% \mathrm{Cl}-0.21$ to 0.14 ) post treatment (see Figure 3 ) or at 5 to 8 month follow-up (SMD $0.11 ; 95 \% \mathrm{Cl}-0.13$ to 0.35 ). However, a large difference was found at 9 to 12 month follow-up (SMD $-0.86 ; 95 \% \mathrm{Cl}-1.50$ to -0.22 ).

Insert Figures 3 about here. 
For secondary outcomes, six studies reported data on depressive symptoms (Buhmann et al., 2016; Hensel-Dittmann et al., 2011; Neuner et al., 2004; Paunovic \& Ost, 2001; Ter Heide et al., 2011, 2016). Data from Neuner et al. (2004) and ter Heide et al. (2016) were reported in a manner that could not be used. We found no difference between conditions (SMD $-0.21 ; 95 \% \mathrm{Cl}-0.59$ to 0.16 ) for the remaining four studies. Two trials (HenselDittmann et al., 2011; ter Heide et al., 2016) investigated effect of intervention on PTSD diagnosis at post intervention. We found no difference (RR $0.98 ; 95 \% \mathrm{Cl} 0.84$ to 1.15 ) between conditions. One trial (Neuner et al., 2004) investigated PTSD diagnosis at 1 year follow up and reported a difference in favour of NET (RR $0.36 ; 95 \% \mathrm{Cl} 0.15$ to 0.87 ) with large reduction in the NET group (29\%) compared with a small reduction in the supportive counselling (79\%) group. All seven trials investigated participant dropout. We found no difference (RR 0.99; 95\% Cl 0.50 to 1.96) between conditions. Using GRADE, we rated the quality of evidence for all findings as low to very low.

\section{Group-based psychotherapy}

Only one study included in this review investigated the effect of group-based psychotherapy. Otto and colleagues (2003) compared combined treatment consisting of a trauma-focused CBT intervention and sertraline, with a condition involving sertraline only in a group of pharmacotherapy-refractory Cambodian refugees. Small to large effects in favour of combined treatment for PTSD severity were reported. No difference in effect for depressive symptoms was found. The study only involved 10 participants and because of the way that the data was reported we were unable to carry out an analysis.

\section{Sub-group analyses}

Eye Movement Desensitisation and Reprocessing (EMDR)

Four studies investigated EMDR with two comparing the psychological intervention to a waitlist control (Acarturk et al., 2015, 2016) and two comparing EMDR versus a stabilisation 
condition (ter Heide et al., 2011, 2016). We found a large effect (SMD $-1.48 ; 95 \% \mathrm{Cl}-1.88$ to -1.09) in favour of EMDR for PTSD severity when compared against an inactive control. No additional follow-up data was available. In an analysis of EMDR versus active control for PTSD symptoms at post-intervention. We found no difference (SMD $-0.29 ; 95 \% \mathrm{Cl}-0.94$ to 0.37) between conditions when EMDR was compared against active control, which was a stabilisation intervention in both studies. No data was available at follow-up. We assessed the quality of evidence for all findings as very low.

\section{Narrative Exposure Therapy (NET)}

Two studies (Neuner et al., 2008, 2010) which compared NET with an inactive control did not report outcome data at post-intervention. An analysis of three studies (Adenauer et al., 2011; Hijazi et al., 2014; Stenmark et al., 2013) comparing NET with a waitlist control found no difference between groups (SMD $-0.80 ; 95 \% \mathrm{Cl}-1.65$ to 0.05$)$ for PTSD severity at postintervention, although there was a trend in favour of NET. Two studies (Neuner et al., 2008, 2010) contributed to an analysis of NET with minimal intervention for PTSD severity at 5-8 months follow-up. We found a moderate effect in favour of NET (SMD $-0.62 ; 95 \% \mathrm{Cl}-0.93$ to -0.32). Three studies (Hensel-Dittmann et al., 2011; Neuner et al., 2008, 2004) compared NET with an active control, stress inoculation therapy, trauma counselling or supportive counselling. We found no difference between conditions at post-intervention (SMD -0.01; $95 \% \mathrm{Cl}-0.25$ to 0.23 ) or $5-8$ month follow-up (SMD $-0.01 ; 95 \% \mathrm{Cl}-0.54$ to 0.53 ). However, a large difference was found at 9 to 12 month follow-up (SMD $-0.86 ; 95 \% \mathrm{Cl}-1.50$ to -0.22 ). We assessed the quality of evidence for all findings as low to very low.

\section{Individual Cognitive Behavioural Therapy (CBT)}

Three studies (Buhmann et al., 2016; Hinton et al., 2004, 2005) compared CBT with a waitlist control. We found no difference (SMD -1.32; $95 \% \mathrm{Cl}-3.17$ to 0.53 ) between conditions. No follow-up data was available. One study ( Paunovic \& Ost, 2001) compared 
CBT with another active psychological intervention. Paunovic \& Ost, (2001) compared CBT with exposure therapy. There was no difference for PTSD severity at post-intervention (MD $0.12 ; 95 \% \mathrm{Cl}-0.76$ to 1.00$)$. Paunovic \& Ost, (2001) or at 5-8 month follow-up (MD 2.40; 95\% Cl -20.31 to 25.11). In a 2x2 design Buhmann et al., 2016 compared CBT as part of a combined treatment of CBT and Sertraline versus Sertraline and versus a waitlist control. We found no difference for either analyses (MD $0.00 ;-0.24$ to 0.24 and $0.00 ; 95 \% \mathrm{Cl}-0.33$ to 0.33 respectively) between conditions. No data was available at follow-up. We assessed the quality of evidence for all findings as very low. 


\section{Discussion}

We included 16 randomised controlled trials (RCTs) with 1,111 participants in this review. In accordance with previous systematic reviews and meta-analyses conducted with asylum seeker and refugee populations (Lambert \& Alhassoon, 2015; Nosè et al., 2017) this study found empirical evidence for the effectiveness of trauma-focused psychological interventions in reducing PTSD symptoms. The data suggests a large effect in favour of trauma-focused psychotherapy (TFP) for our primary outcome of PTSD severity and reduced frequency of PTSD diagnosis when compared with inactive controls. Effects were similar for depression and we found no difference for participant drop-out. We found some evidence in favour of TFP when comparing TFP with active controls based on data from two NET studies. We were able to undertake some subgroup analyses of specific interventions, although the number of available studies contributing to these analyses was small. We found some evidence for both EMDR and NET. The evidence for EMDR compared against waitlist controls was from two small studies in comparison to waitlist controls. The data showed a large effect in favour of EMDR, which suggests that EMDR is a potentially promising intervention in this population. However, these studies did not undertake long-term follow-up and we did not find a difference of effect in an analysis comparing EMDR with stabilisation as an active control. Both analyses need to be interpreted with some caution given the small number of trials and relatively small sample sizes. Positive findings for NET in long term follow-up are consistent with those reported in other refugee reviews (Crumlish \& O Rourke, 2010; Nosè et a, 2017; Robjant \& Fazel, 2010). We note that these studies were all conducted by the same group of researchers who acknowledged therapist allegiance to NET. We did not find evidence to support the use of other CBT based approaches. No trials investigated more established forms of TFP such as Prolonged Exposure, CPT or CT and like Nickerson et al, (2011) we did not find any RCTs of multimodal interventions for PTSD. 
In common with many reviews of this kind (e.g. Bisson et al., 2015; Roberts et al., 2015) there was a high degree of clinical and methodological divergence amongst included studies in terms of interventions delivery, study population features and study features. By including RCTs only in this review, we sought to strengthen the evidence base from which we have made conclusions by reducing risk of bias from unobserved heterogeneity. We used robust methods for analysing the methodological quality of included studies and the quality of evidence of pooled data, in line with Cochrane (Higgins \& Green, 2011), SURE (Specialist Unit for Review Evidence (SURE), 2013) and GRADE systems (Guyatt et al., 2013; Guyatt et al., 2011). GRADE judgements were influenced by the heterogeneity issues we have described, limited study numbers and small sample sizes. We rated the quality of all evidence obtained as low to very low, therefore, these findings need to be interpreted with caution and may be liable to change as further evidence accumulates. On first sight, these GRADE ratings may appear somewhat disheartening. However, we feel that it is important to recognise the particularly challenging contexts within which many of these studies have been undertaken and the complexity of need of the target population (Carlsson et al., 2014). It is also important to recognise that low GRADE judgments affect many reviews of psychological interventions, including other trauma and PTSD populations (Bisson et al., 2013; Roberts et al., 2015). The International Society for Traumatic Stress Studies (ISTSS) has recently considered this issue in developing the forthcoming ISTSS Treatment Guidelines (https://www.istss.org/treating-trauma/new-istss-guidelines.aspx). They have developed draft recommendation settings based on four possible recommendations ("strong", "standard", "intervention with promise" and insufficient evidence to recommend), which takes into account size of effect, GRADE judgement and other contextual factors (Bisson, Berliner, \& Monson, 2017). Although the current guideline committee is not considering recommendations for specific populations, it is likely that trauma focused psychological therapy, EMDR and NET for refugees and asylum seekers would all reach an evidence threshold to receive positive recommendations based on these criteria. 
Meta analyses focusing on the available evidence for a specific sub-population will inevitably suffer reduced statistical power in comparison to reviews undertaken with wider populations which will normally reduce confidence about outcomes. Findings therefore need to be judged in terms of consistency with the broader evidence base. Given the particular challenges of undertaking high quality studies with refugee populations, we feel that our findings are encouraging. They are consistent with findings from a recent review of psychological interventions for survivors of mass violence in low and middle-income countries (LMICs) (Morina, Malek, Nickerson \& Bryant, 2017), a review of interventions for torture survivors (Patel et al, 2014) and they are broadly consistent with the established evidence base on psychological interventions for PTSD (Bisson et al., 2013). These findings therefore suggest that trauma focused psychological therapies can be effective in improving symptoms for refugees and asylum seekers with PTSD.

Our review used stringent methodology to analyse the evidence base but has some limitations. Our search strategy only identified studies published in English and as such we may have missed robust studies published in other languages. We made efforts to obtain missing data deemed important for inclusion in meta-analyses, but we did not receive responses from some authors. Meta-analysis only rarely involves synthesis of data from identical studies (Borenstein, Hedges, Higgins, \& Rothstein, 2011). We attempted to group studies together in a way that was logical and clinically meaningful. However, in view of the level of clinical heterogeneity within comparisons we decided to use random effects analyses throughout. The small pool of studies limited the number of analyses that could be undertaken. For instance, we were unable to evaluate the effects of intervention for asylum seeker and refugee samples separately or explore difference between studies undertaken in high-income and low-income countries. We were not able to investigate for publication bias.

Findings from this review have important implications for clinical practice and research which can be used to support the creation of policy and guidelines. This review builds on the 
existing evidence base for psychological interventions with refugee and asylum seeker populations and provides evidence for the benefits of providing trauma-focused therapy for refugees and asylum seekers with PTSD and preliminary evidence for EMDR and NET.

We found the current evidence base to be limited by a small number of RCTs of variable methodical quality. Few trials compared the main intervention to an established, alternative psychological intervention. Only one small trial investigated a group-format intervention. However, despite the particular challenges of undertaking clinical trials with refugee populations, including studies in refugee camps in developing countries, study authors have generally demonstrated that it is possible to conduct such trials whilst adhering to most conventional trial standards. Larger, more robust trials are needed to replicate findings and to support the development of new interventions. Carlsson et al (2014) and Nickerson, et al (2011) have argued for the benefits of an iterative process of intervention development and evaluation within specialist refugee services. Direct comparisons between psychological interventions will help to determine efficacy. We found trials investigating a narrow range of interventions: EMDR, CBT and NET. It is not clear that refugees and asylum seekers would benefit from other established evidence-based treatments available in many developed countries. However, we note there are a number of large studies have reported positive outcomes in trials aimed at evaluating interventions such as Behavioural Activation (BA), Cognitive Processing Therapy (CPT) and Common Elements Treatment Approach (CETA) (Bass, Annan, Mclvor Murray, Kaysen, Griffiths, et al, 2013; Bass, Murray, Mohammed, Bunn, Gorman, Ahmed et al, 2016; Betancourt, McBain, Newnham, Akinsulure-Smith, Brennan, Weitz et al, 2014; Bolton, Bass, Zangana, Kamal, Murray, Kaysen, et al, 2014; Bolton, Lee, Haroz, Murray, Dorsey, Robinson et al, 2014) amongst trauma and conflict exposed populations in a number of LMICs. This evidence is therefore pointing to the fact that many established psychological interventions are potentially applicable to refugees and other conflict exposed individuals from LMICs. We agree with Nickerson and colleagues that it is particularly important for Western treatment models to give careful attention to culturally 
specific adaptations to ensure acceptability and appropriateness for refugees. There is also a need for trials to undertake further investigation of non-trauma-focused interventions, such as stress management and mindfulness (Carlsson et al., 2014). Trials should be conducted by researchers who are independent from the mode of therapy to reduce possible researcher bias due to therapy-allegiance.

Few studies in our review investigated longer term effects. Longitudinal trials are needed to enhance our understanding of the sustainability of psychological interventions. Our review shows that individual trauma-focused interventions currently dominate research trials. Considering the complexity of contextual factors affecting outcomes, it seems important that trials should not limit their attention to individual trauma focused interventions but should consider the evaluation of social, familial, human rights and welfare interventions as well ( $\mathrm{N}$ Patel et al., 2016; Steel, Bateman Steel, \& Silove, 2009).

In summary, this review provides support for the use of psychological interventions for PTSD in asylum seeker and refugee populations. However, it also highlights substantial gaps in our understanding of how best to support refugees and asylum seekers with complex difficulties. Future studies should aim to robustly evaluate the efficacies of treatment approaches which will be essential for informing clinical practice guidelines and enhancing psychological wellbeing.

\section{Role of funding sources}

This study was not directly funded but was undertaken whilst CT was undertaking a doctoral dissertation and was supported by South Wales Doctoral Programme in Clinical Psychology, Cardiff \& Vale University Health Board and the Division of Psychological Medicine and Clinical Neurosciences, Cardiff University School of Medicine. These organizations had no 
role in the study design, collection, analysis or interpretation of the data, writing the manuscript, or the decision to submit the paper for publication.

\section{Author contributions}

CT and AV designed the study and wrote the protocol and conducted literature searches and acquisition of data. NPR offered supervision. CT and NPR conducted the statistical analyses. CT wrote the first draft of the manuscript and all authors contributed to and have approved the final manuscript.

\section{Conflict of interest}

None of the authors have any conflicts of interest to disclose.

\section{Acknowledgements}

With thanks to Dr Dougal Hare for assisting with the management of the project. Also to study authors Dr Håkon Stenmark, Dr Cerin Acartürk, Dr Jackie June ter Heide, Dr Frank Neuner and Dr Mark Lumley for providing information or data for the meta-analysis. 


\section{References}

Acarturk, C., Konuk, E., Cetinkaya, M., Senay, I., Sijbrandij, M., Cuijpers, P., \& Aker, T. (2015). Emdr for syrian refugees with posttraumatic stress disorder symptoms: Results of a pilot randomized controlled trial. European Journal of Psychotraumatology, 6, 1-9. https://doi.org/10.3402/ejpt.v6.27414

Acarturk, C., Konuk, E., Cetinkaya, M., Senay, I., Sijbrandij, M., Gulen, B., ... Xu, R. (2016). The efficacy of eye movement desensitization and reprocessing for post-traumatic stress disorder and depression among Syrian refugees: results of a randomized controlled trial. Psychological Medicine, 46(12), 2583-2593. https://doi.org/10.1017/S0033291716001070

Adenauer, H., Catani, C., Gola, H., Keil, J., Ruf, M., Schauer, M., \& Neuner, F. (2011). Narrative exposure therapy for PTSD increases top-down processing of aversive stimuli - evidence from a randomized controlled treatment trial. BMC Neuroscience, 12(1), 127. https://doi.org/10.1186/1471-2202-12-127

American Psychiatric Association (Ed.). (1980). Diagnostic and statistical manual of mental disorders ((3rd ed.)). Washington, DC.

Bass, J.K., Annan, J., Mclvor Murray, S., Kaysen, D., Griffiths, S., ... Bolton, P.A. (2013). Controlled Trial of Psychotherapy for Congolese Survivors of Sexual Violence. New England Journal of Medicine, 368 (23) 2182-2191.

Bass J., Murray S.M., Mohammed T.A., Bunn M., Gorman W., Ahmed A.M.A., Murray, L. Bolton, P. (2016). A randomized controlled trial of a traumainformed support, skills, and psychoeducation intervention for survivors of torture and related trauma in Kurdistan, Northern Iraq. Global Health Science and Practice, 4(3), 452-466. http:/dx.doi.org/10.9745/GHSP-D-16-00017

Betancourt, T.S., McBain, R. Newnham, E.A., Akinsulure-Smith, A.M., Brennan, R.T., ... 
Hansen N.B. (2014). A Behavioral Intervention for War-Affected Youth in Sierra Leone:

A Randomized Controlled Trial. Journal of the American Academy of Child \&

Adolescent Psychiatry, 53(12), 1288-1297. https://doi.org/10.1016/j.jaac.2014.09.011

Bisson, J. I., Berliner, L., \& Monson, C. (2017). ISTSS Treatment Guidlines. Paper presented at the International Society for Traumatic Stress Studies Annual Meeting, Chicago, November 2017.

Bisson, J. I., Cosgrove, S., Lewis, C., \& Roberts, N. P. (2015). Post-traumatic stress disorder. BMJ, 351. https://doi.org/doi: 10.1136/bmj.h6161

Bisson, J. I., Roberts, N. P., Andrew, M., Cooper, R., \& Lewis, C. (2013). Psychological therapies for chronic post-traumatic stress disorder (PTSD) in adults. In J. I. Bisson (Ed.), Cochrane Database of Systematic Reviews. Chichester, UK: John Wiley \& Sons, Ltd. https://doi.org/10.1002/14651858.CD003388.pub4

Blake, D. D., Weathers, F. W., Nagy, L. M., Kaloupek, D. G., Gusman, F. D., Charney, D. S., \& Keane, T. M. (1995). The development of a Clinician-Administered PTSD Scale. Journal of Traumatic Stress, 8(1), 75-90. https://doi.org/DOI: 10.1002/jts.2490080106

Bogic, M., Njoku, A., Priebe, S., Fabrega, H., Parron, D., \& Good, B. (2015). Long-term mental health of war-refugees: a systematic literature review. BMC International Health and Human Rights, 15(1), 29. https://doi.org/10.1186/s12914-015-0064-9

Bolton, P., Bass, J.K., Zangana, G.A.S., Kamal, T., Murray, S.M., ... Rosenblum, M. (2014). A randomized controlled trial of mental health interventions for survivors of systematic violence in Kurdistan, Northern Iraq. BMC Psychiatry, 14:360 https://doi.org/10.1186/s12888-014-0360-2

Bolton, P., Lee, C., Haroz, E.E., Murray, L., Dorsey, S., Robinson, C., Ugato, A.M. \& Bass, J.K. (2014). A Transdiagnostic Community-Based Mental Health Treatment for Comorbid Disorders: Development and Outcomes of a Randomized Controlled Trial among Burmese Refugees in Thailand. PLOS Medicine, 11(11), e1001757. doi:10.1371/journal.pmed. 1001757 
Borenstein, M., Hedges, L. V., Higgins, J. P. T., \& Rothstein, H. (2011). Introduction to MetaAnalysis. Wiley.

Buhmann, C. B., Nordentoft, M., Ekstroem, M., Carlsson, J., \& Mortensen, E. L. (2016). The effect of flexible cognitive-behavioural therapy and medical treatment, including antidepressants on post-traumatic stress disorder and depression in traumatised refugees: Pragmatic randomised controlled clinical trial. British Journal of Psychiatry, 208(3), 252-259. https://doi.org/10.1192/bjp.bp.114.150961

Burnett, A., \& Peel, M. (2001). Asylum seekers and refugees in Britain. The health of survivors of torture and organised violence. BMJ (Clinical Research Ed.), 322(7286), 606-9. https://doi.org/doi: https://doi.org/10.1136/bmj.322.7286.606

Carlsson, J., Sonne, C., \& Silove, D. (2014). From Pioneers to Scientists. The Journal of Nervous and Mental Disease, 202(9), 630-637.

https://doi.org/10.1097/NMD.0000000000000175

Crumlish, N., \& O'Rourke, K. (2010). A Systematic Review of Treatments for Post-Traumatic Stress Disorder Among Refugees and Asylum-Seekers. The Journal of Nervous and Mental Disease, 198(4), 237-251. https://doi.org/10.1097/NMD.0b013e3181d61258

Ehlers, A., \& Clark, D. M. (2000). A cognitive model of posttraumatic stress disorder. Behaviour Research and Therapy, 38(4), 319-345. https://doi.org/10.1016/S00057967(99)00123-0

Ehring, T., Welboren, R., Morina, N., Wicherts, J. M., Freitag, J., \& Emmelkamp, P. M. G. (2014). Meta-analysis of psychological treatments for posttraumatic stress disorder in adult survivors of childhood abuse. Clinical Psychology Review, 34(8), 645-657. https://doi.org/10.1016/j.cpr.2014.10.004

Fazel, M., Wheeler, J., \& Danesh, J. (2005). Prevalence of serious mental disorder in 7000 refugees resettled in western countries: a systematic review. The Lancet, 365(9467), 1309-1314. https://doi.org/10.1016/S0140-6736(05)61027-6 
Foa, E. B., Cashman, L., Jaycox, L., \& Perry, K. (1997). The validation of a self-report measure of posttraumatic stress disorder: The Posttraumatic Diagnostic Scale. Psychological Assessment, 9(4), 445-451. https://doi.org/10.1037/1040-3590.9.4.445

Foa, E. B., Hembree, E. A., \& Rothbaum, B. O. (2007). Prolonged exposure therapy for PTSD : emotional processing of traumatic experiences : therapist guide. Oxford University Press.

Guyatt, G. H., Oxman, A. D., Schünemann, H. J., Tugwell, P., \& Knottnerus, A. (2011). GRADE guidelines: A new series of articles in the Journal of Clinical Epidemiology. Journal of Clinical Epidemiology, 64(4), 380-382. https://doi.org/10.1016/j.jclinepi.2010.09.011

Guyatt, G., Oxman, A. D., Sultan, S., Brozek, J., Glasziou, P., Alonso-Coello, P., ... Schünemann, H. J. (2013). GRADE guidelines: 11. Making an overall rating of confidence in effect estimates for a single outcome and for all outcomes. Journal of Clinical Epidemiology, 66(2), 151-157. https://doi.org/10.1016/j.jclinepi.2012.01.006 Hedges, L. V. (1981). Distribution Theory for Glass's Estimator of Effect size and Related Estimators. Journal of Educational and Behavioral Statistics, 6(2), 107-128. https://doi.org/10.3102/10769986006002107

Hensel-Dittmann, D., Schauer, M., Ruf, M., Catani, C., Odenwald, M., Elbert, T., \& Neuner, F. (2011). Treatment of traumatized victims of war and torture: a randomized controlled comparison of narrative exposure therapy and stress inoculation training. Psychotherapy and Psychosomatics, 80(6), 345-52. https://doi.org/10.1159/000327253 Higgins, J., \& Green, S. (2011). Cochrane handbook for systematic reviews of interventions: Version 5.3.5. The Cochrane Collaboration. Retrieved from http://handbook.cochrane.org/

Hijazi, A. M., Lumley, M. A., Ziadni, M. S., Haddad, L., Rapport, L. J., \& Arnetz, B. B. (2014). Brief Narrative Exposure Therapy for Posttraumatic Stress in Iraqi Refugees: A 
Preliminary Randomized Clinical Trial. Journal of Traumatic Stress, 27(3), 314-322. https://doi.org/10.1002/jts.21922

Hinton, D. E., Chhean, D., Pich, V., Safren, S., Hofmann, S. G., \& Pollack, M. H. (2005). A randomized controlled trial of cognitive-behavior therapy for Cambodian refugees with treatment-resistant PTSD and panic attacks: A cross-over design. Journal of Traumatic Stress, 18(6), 617-629. https://doi.org/10.1002/jts.20070

Hinton, D. E., Pham, T., Tran, M., Safren, S., Otto, M. W., \& Pollack, M. H. (2004). CBT for Vietnamese RefugeesWith Treatment-Resistant PTSD ans Panic Attacks: A Pilot Study. Journal of Traumatic Stress, 17(5), 429-433.

https://doi.org/10.1023/B:JOTS.0000048956.03529.fa

Hollander, A.-C., Dal, H., Lewis, G., Magnusson, C., Kirkbride, J. B., \& Dalman, C. (2016). Refugee migration and risk of schizophrenia and other non-affective psychoses: cohort study of 1.3 million people in Sweden. BMJ, 352. https://doi.org/doi: 10.1136/bmj.i1030 Jaranson, J. M., Jacobs, U., Kinzie, D., \& Quiroga, J. (2006). Standard therapy for all torture survivors?: rapid response to M. Basoglu. Rehabilitation of traumatised refugees and survivors of torture. BMJ (Clinical Research Ed.), 333(7581), 1230-1. https://doi.org/10.1136/bmj.39036.739236.43

Kalt, A., Hossain, M., Kiss, L., \& Zimmerman, C. (2013). Asylum seekers, violence and health: a systematic review of research in high-income host countries. American Journal of Public Health, 103(3), e30-42. https://doi.org/10.2105/AJPH.2012.301136

Lambert, J. E., \& Alhassoon, O. M. (2015). Trauma-focused therapy for refugees: Metaanalytic findings. Journal of Counseling Psychology, 62(1), 28-37.

https://doi.org/10.1037/cou0000048

Langlois, E., Haines, A., Tomson, G. \& Ghaffar, A. (2016). Refugees: towards better access to health-care services. Lancet, 387(10016), 319-321. doi:10.1016/S01406736(16)00101-X 
Morina, M., Malek, M., Nickerson, A., \& Bryant, R. (2017). Meta-analysis of interventions for posttraumatic stress disorder and depression in adult survivors of mass violence in low- and middle-income countries. Depression \& Anxiety, 34(8), 679-691.

Neuner, F., Kurreck, S., Ruf, M., Odenwald, M., \& Schauer, M. (2010). Can Asylum-Seekers with Posttraumatic Stress Disorder Be Successfully Treated? A Randomized Controlled Pilot Study Can Asylum-Seekers with Posttraumatic Stress Disorder Be Successfully Treated? A Randomized Controlled Pilot Study. Cognitive Behaviour Therapy, 6073(September 2016), 81-91. https://doi.org/10.1080/16506070903121042

Neuner, F., Onyut, P. L., Ertl, V., Odenwald, M., Schauer, E., \& Elbert, T. (2008). Treatment of Posttraumatic Stress Disorder by Trained Lay Counselors in an African Refugee Settlement : A Randomized Controlled Trial. Journal of Consulting and Clinical Psychology, 76(4), 686-694. https://doi.org/10.1037/0022-006X.76.4.686

Neuner, F., Schauer, M., Klaschik, C., Karunakara, U., \& Elbert, T. (2004). A comparison of narrative exposure therapy, supportive counseling, and psychoeducation for treating posttraumatic stress disorder in an african refugee settlement. Journal of Consulting and Clinical Psychology, 72(4), 579-587. https://doi.org/10.1037/0022-006X.72.4.579

Nickerson, A., Bryant, R.A., Silove, D., \& Steel, Z. (2011). A critical review of psychological treatments of posttraumatic stress disorder in refugees. Clinical Psychology Review, 31(3), 399-417. https://doi.10.1016/j.cpr.2010.10.004

Nosè, M., Ballette, F., Bighelli, I., Turrini, G., Purgato, M., Tol, W., ... Barbui, C. (2017). Psychosocial interventions for post-traumatic stress disorder in refugees and asylum seekers resettled in high-income countries: Systematic review and meta-analysis. PloS One, 12(2), e0171030. https://doi.org/10.1371/journal.pone.0171030

Otto, M. W., Hinton, D. E., Korbly, N. B., Chea, A., Ba, P., Gershuny, B. S., \& Pollack, M. H. (2003). Treatment of pharmacotherapy-refractory posttraumatic stress disorder among Cambodian refugees : a pilot study of combination treatment with cognitive-behavior therapy vs sertraline alone. Behaviour Research and Therapy, 41(41), 1271-1276. 
https://doi.org/10.1016/S0005-7967(03)00032-9

Patel, N., Kellezi, B., \& Williams, A. C. de C. (2014). Psychological, social and welfare interventions for psychological health and well-being of torture survivors. In A. C. de C. Williams (Ed.), Cochrane Database of Systematic Reviews. Chichester, UK: John Wiley \& Sons, Ltd. https://doi.org/10.1002/14651858.CD009317.pub2

Patel, N., Williams, A., \& Kellezi, B. (2016). Reviewing outcomes of psychological interventions with torture survivors: conceptual, methodological and ethical issues. Torture. Retrieved from http://www.corteidh.or.cr/tablas/r34985.pdf

Paunovic, N., \& Ost, L.-G. (2001). Cognitive-behavior therapy vs exposure therapy in the treatment of PTSD in refugees. Behaviour Research and Therapy, 39(39), 1183-1197. https://doi.org/https://doi.org/10.1016/S0005-7967(00)00093-0

Priebe S, Giacco D, El-Nagib R. Public health aspects of mental health among migrants and refugees: a review of the evidence on mental health care for refugees, asylum seekers and irregular migrants in the WHO European Region. Copenhagen: WHO Regional Office for Europe; 2016 (Health Evidence Network (HEN) Synthesis Report 47).

Resick, P. A., \& Schnicke, M. K. (1993). Cognitive processing therapy for rape victims : a treatment manual. Sage Publications.

Review Manager (RevMan) [Computer program] Copenhagen: The Nordic Centre. (2011). The Cochrane Collaboration. Retrieved from http://tech.cochrane.org/\%0Arevman/download

Roberts, N. P., Roberts, P. A., Jones, N., \& Bisson, J. I. (2015). Psychological interventions for post-traumatic stress disorder and comorbid substance use disorder: A systematic review and meta-analysis. Clinical Psychology Review, 38, 25-38. https://doi.org/10.1016/j.cpr.2015.02.007

Robjant, K., \& Fazel, M. (2010). The emerging evidence for Narrative Exposure Therapy: A review. Clinical Psychology Review, 30(8), 1030-1039. 
https://doi.org/10.1016/j.cpr.2010.07.004

Schauer, M., Elbert, T., \& Neuner, F. (2011). Narrative exposure therapy : a short-term treatment for traumatic stress disorders. Hogrefe.

Specialist Unit for Review Evidence (SURE). (2013). Questions to assist with the critical appraisal of randomised controlled trials and other experimental studies. Available at http://www.cardiff.ac.uk/insrv/libraries/sure/doc/SURE_RCT_Checklist_2013.pdf. Retrieved from http://www.cardiff.ac.uk/_data/assets/pdf_file/0006/212766/SURE_RCTs-and-otherexperimental-studies_Checklist_2015-update.pdf

Steel, Z., Bateman Steel, C. R., \& Silove, D. (2009). Human rights and the trauma model: Genuine partners or uneasy allies? Journal of Traumatic Stress, 22(5), 358-365. https://doi.org/10.1002/jts.20449

Steel, Z., Chey, T., Silove, D., Marnane, C., Bryant, R. A., \& van Ommeren, M. (2009). Association of Torture and Other Potentially Traumatic Events With Mental Health Outcomes Among Populations Exposed to Mass Conflict and Displacement. JAMA, 302(5), 537. https://doi.org/10.1001/jama.2009.1132

Stenmark, H., Catani, C., Neuner, F., Elbert, T., \& Holen, A. (2013). Treating PTSD in refugees and asylum seekers within the general health care system. A randomized controlled multicenter study. Behaviour Research and Therapy (Vol. 51). https://doi.org/10.1016/j.brat.2013.07.002

Ter Heide, F. J. J., Mooren, T. M., Kleijn, W., de Jongh, A., \& Kleber, R. J. (2011). EMDR versus stabilisation in traumatised asylum seekers and refugees: results of a pilot study. European Journal of Psychotraumatology, 2, 1-11. https://doi.org/10.3402/ejpt.v2i0.5881

Ter Heide, F. J. J., Mooren, T. M., Van De Schoot, R., De Jongh, A., \& Kleber, R. J. (2016). Eye movement desensitisation and reprocessing therapy v. stabilisation as usual for 
refugees: Randomised controlled trial. British Journal of Psychiatry, 209(4), 311-318. https://doi.org/10.1192/bjp.bp.115.167775

Turner, S. W., \& Herlihy, J. (2009). Working with refugees and asylum seekers. Psychiatry, 8(8), 322-324. https://doi.org/10.1016/J.MPPSY.2009.05.002

UN General Assembly. (1951). UNHCR - Protocol Relating to the Status of Refugees.

Retrieved April 17, 2017, from http://www.unhcr.org/3b66c2aa10.html

UNHCR. (2016). Global Trends: Forced Displacement in 2015. Retrieved from

https://s3.amazonaws.com/unhcrsharedmedia/2016/2016-06-20-global-trends/2016-0614-Global-Trends-2015.pdf 
Databases searched using ProQuest

(including selected databases ASSIA,

IBSS, PILOTS), Web of Science,

Psychlnfo, CENTRAL, CDSR and

reference lists of reviews and included studies $(\mathrm{n}=864)$

After title and abstract review with study exclusion for:

- Study design is non-experimental (no randomisation or no control)

- Study's primary focus is not to investigate clinical efficacy of psychological intervention for PTSD

$(n=141) 384$ excluded

After full paper review with study exclusion for previous exclusion criteria and:

- Study is not a primary research paper

- Less than $80 \%$ of participants are an asylum seeker or refugee

- Less than $80 \%$ of participants have a PTSD diagnosis, by a clinician via a diagnosis using a structured interview or a validated self-report measure

- Not all participants aged 18 or over

$(n=16) 118$ excluded

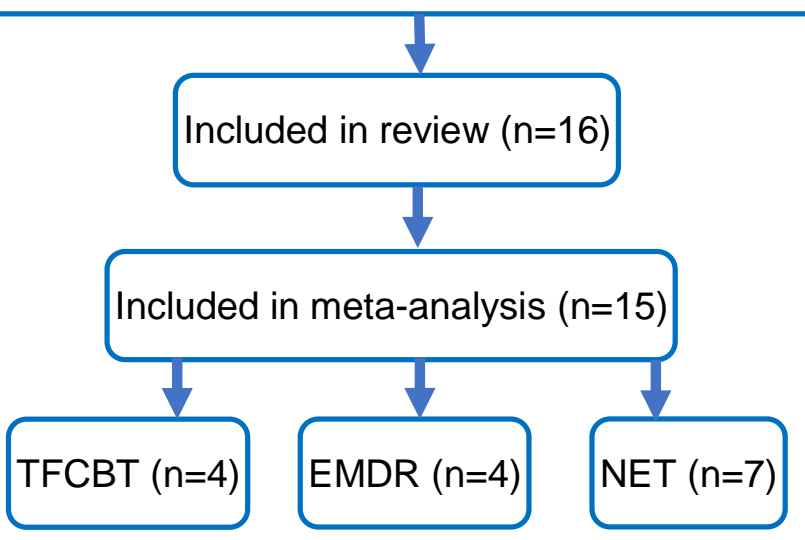

Figure 1. PRISMA flow diagram of search methodology 
Table 1. Characteristics of included randomised controlled trials

\begin{tabular}{|c|c|c|c|c|c|c|}
\hline $\begin{array}{l}\text { Authors, Year, Country of } \\
\text { study }\end{array}$ & Comparison & $\begin{array}{l}\text { Sample size, } \\
\text { Gender \& Age }\end{array}$ & $\begin{array}{l}\text { Participants' } \\
\text { Country of } \\
\text { Origin } \\
\end{array}$ & PTSD diagnostic instrument & PTS Measures & Other Outcome Measures \\
\hline $\begin{array}{l}\text { Acarturk, Konuk, } \\
\text { Cetinkaya, Senay, } \\
\text { Sijbrandij, Cuijpers \& Aker } \\
\text { (2015) Turkey }\end{array}$ & EMDR, WL & $\begin{array}{l}N=29,7 \text { male, } \\
22 \text { female, } \\
\text { Mean age }=36\end{array}$ & Syria & $\begin{array}{l}\text { Cut-off score }(\geq 33) \text { used on } \\
\text { Impact of Event Scale - Revised } \\
\text { (IES-R) (Creamer \& Falilla, 2002) }\end{array}$ & IES-R (Weiss \& Marmar, 1997) & $\begin{array}{l}\text { Beck Depression Inventory (Beck, Steer \& } \\
\text { Brown, 1996) Arabic version (Ghareeb, 2000) }\end{array}$ \\
\hline $\begin{array}{l}\text { Acarturk, Konuk, } \\
\text { Cetinkaya, Senay, } \\
\text { Sijbrandij, Gulen } \\
\text { \&Cuijpers (2016) Turkey }\end{array}$ & EMDR, WL & $\begin{array}{l}\mathrm{N}=70,25 \\
\text { males, } 45 \\
\text { females, Mean } \\
\text { age }=33\end{array}$ & Syria & $\begin{array}{l}\text { Diagnosis (DSM-IV) using the } \\
\text { Mini-International } \\
\text { Neuropsychiatric Interview Plus } \\
\text { (M.I.N.I. PLUS) (Sheehan et al. } \\
\text { 1998) }\end{array}$ & $\begin{array}{l}\text { Harvard Trauma Questionnaire } \\
\text { (HTQ) (Mollica et al., 1992) } \\
\text { Arabic version (Shoeb et al., } \\
\text { 2007), Impact of Event Scale- } \\
\text { Revised (IES-R) (Weiss \& Marmar, } \\
\text { 1997) }\end{array}$ & $\begin{array}{l}\text { Beck Depression Inventory-II (BDI-II) (Beck et } \\
\text { al, (1996) Arabic version (Ghareeb, 2000), } \\
\text { Hopkins Symptoms Checklist-25 (HSC-25) } \\
\text { (Mollica et al.,2004) Arabic version (Kobeissi et } \\
\text { al., 2011) }\end{array}$ \\
\hline $\begin{array}{l}\text { Adenauer, Catani, Gola, } \\
\text { Keil, Ruf, Schauer \& } \\
\text { Neuner (2011) Germany }\end{array}$ & NET, WL & $\begin{array}{l}\mathrm{N}=34,19 \text { male, } \\
15 \text { female, } \\
\text { Mean age }=35\end{array}$ & $\begin{array}{l}\text { Middle East, } \\
\text { Central East, } \\
\text { The Balkans, } \\
\text { Africa }\end{array}$ & $\begin{array}{l}\text { Diagnosis using DSM-IV (APA, } \\
\text { 1994) criteria with Clinician } \\
\text { Administered PTSD Scale (CAPS) } \\
\text { (Blake et al., 1995) }\end{array}$ & $\begin{array}{l}\text { Clinician Administered PTSD } \\
\text { Scale (CAPS) (Blake et al., 1995) }\end{array}$ & $\begin{array}{l}\text { Vivo checklist of war, detentions and violent } \\
\text { events, Mini-International Neuropsychiatric } \\
\text { Interview Plus (M.I.N.I. PLUS) (Sheehan et al. } \\
\text { 1998), Hamilton Depression Rating Scale } \\
\text { (HDRS) (Williams, 1988) }\end{array}$ \\
\hline $\begin{array}{l}\text { Buhmann, Nordentoft, } \\
\text { Ekstrom, Carlsson \& } \\
{\text { Mortensen }(2016)^{\text {a }}}^{\text {Denmark }}\end{array}$ & $\begin{array}{l}\text { CBT (inc exposure), } \\
\text { Antidepressants } \\
\text { (Sertraline and } \\
\text { Mianserin), CBT (inc } \\
\text { exposure)+ } \\
\text { Antidepressants } \\
\text { (Sertraline and } \\
\text { Mianserin), WL }\end{array}$ & $\begin{array}{l}\mathrm{N}=217,128 \\
\text { males, } 89 \\
\text { females, Mean } \\
\text { age }=45\end{array}$ & $\begin{array}{l}\text { Iraq, Iran, } \\
\text { Lebanon, Ex- } \\
\text { Yugoslavia, } \\
\text { Angola }\end{array}$ & $\begin{array}{l}\text { Diagnosis using ICD-10 (WHO, } \\
\text { 1993) using the Harvard Trauma } \\
\text { Questionnaire (HTQ) using cut- } \\
\text { off score of } 2.5\end{array}$ & $\begin{array}{l}\text { Harvard Trauma Questionnaire } \\
\text { (HTQ) (Mollica et al., 1992, } \\
\text { 1996a) (language versions by } \\
\text { Kleijn et al., 2001) }\end{array}$ & $\begin{array}{l}\text { Hopkins Symptom Checklist (HSCL-25) (Mollica } \\
\text { et al., 1987, 1996b), Symptom Checklist-90 } \\
\text { (SCL-90), Hamilton Rating Scale for Depression } \\
\text { (HRSD) (Hamilton, 1960) and for Anxiety } \\
\text { (HSRA) (Hamilton, 1959), Visual Analogue Pain } \\
\text { Scales (VAS) (Olsen et al., 2007), Sheehan } \\
\text { Disability Scale (SDS) (Sheehan \& Sheehan, } \\
\text { 2008), World Health Organisation-Five Well- } \\
\text { being Index (WHO-5). }\end{array}$ \\
\hline $\begin{array}{l}\text { Hensel-Dittmann, } \\
\text { Schauer, Ruf, Catani, } \\
\text { Odenwald, Elbert \& } \\
\text { Neuner (2011) Germany }\end{array}$ & NET, SIT & $\begin{array}{l}\mathrm{N}=28, \text { gender } \\
\text { and age not } \\
\text { specified }\end{array}$ & Not specified & $\begin{array}{l}\text { Diagnosis using DSM-IV (APA, } \\
\text { 1994) criteria with Clinician } \\
\text { Administered PTSD Scale (CAPS) } \\
\text { (Blake et al., 1995) }\end{array}$ & $\begin{array}{l}\text { Clinician Administered PTSD } \\
\text { Scale (CAPS) (Blake et al., 1995) }\end{array}$ & $\begin{array}{l}\text { Vivo checklist of war, detentions and violent } \\
\text { events, Mini-International Neuropsychiatric } \\
\text { Interview Plus (M.I.N.I. PLUS) (Sheehan et al. } \\
\text { 1998), Hamilton Depression Scale (HAM-D) } \\
\text { (Hamilton, 1960,1967) }\end{array}$ \\
\hline $\begin{array}{l}\text { Hijazi, Lumley, Ziadni, } \\
\text { Haddad, Rapport \& } \\
\text { Arnetz (2014) USA }\end{array}$ & NET, WL & $\begin{array}{l}\mathrm{N}=63,28 \\
\text { males, } 35 \\
\text { females, Mean } \\
\text { age }=48\end{array}$ & Iraq & $\begin{array}{l}\text { Harvard Trauma Questionnaire } \\
\text { (HTQ) (Mollica et al., 1992, } \\
\text { 1996a) }\end{array}$ & $\begin{array}{l}\text { Harvard Trauma Questionnaire } \\
\text { (HTQ) (Mollica et al., 1992, } \\
\text { 1996a) }\end{array}$ & $\begin{array}{l}\text { Posttraumatic Growth Inventory (PTGI) } \\
\text { (Tedeschi \& Calhoun, 1996), World Health } \\
\text { Organisation Well-being Index (WHO-5) Arabic } \\
\text { translation (Bech, 1998), Beck Depression } \\
\text { Inventory-II (BDI-II) (Beck et al., 1996), Patient } \\
\text { Health Questionnaire (PHQ-15) (Kroenke et } \\
\text { al., 2002), A satisfaction with and benefit from } \\
\text { treatment measure }\end{array}$ \\
\hline
\end{tabular}


Hinton, Chhean, Pich, Safren, Hoffman \& Pollack (2005) USA

Hinton, Pham, Tran, Safren, Otto \& Pollack (2004) USA

Neuner, Schauer,

Klaschik, Karunakara \&

Elbert (2004) Uganda

NET, Supportive

counselling,

Psychoeducation

males, 26

females, Mean

age $=33$

Neuner, Onyut, Ertl,

Odenwald, Schauer \&

Elbert (2008) Uganda

Neuner, Kurreck, Ruf,

Odenwald, Elbert \&

Schauer (2010) Germany

Otto, Hinton, Korbly

Chea, Phalnarith,

Gershuny \& Pollack

(2003) USA

Paunovic \& Ost (2001)

Sweden

$N=12$, gender and age not specified

NET, TC, MG

$N=277,135$

$\mathrm{N}=277,135$

females, Mea

age $=35$

NET, TAU

$\mathrm{N}=32,22$

males, 10

females, Mean

age $=31$

CBT (inc exposure,

group format),

Antidepressant

(Sertraline)

CBT (inc exposure)

Exposure therapy
1 Disorders (SCID-I) Module PTSD
$\mathrm{N}=10,0$ males,

10 females,

Mean age $=47$

$\mathrm{N}=20,17$

males, 3

females, Mean

age 38
Diagnosis using Structured

Clinical Interview for DSM-IV Axis

(First et al., 1995)

Clinician-Administered PTSD

Scale (CAPS) (Weathers et al., 2001)

Diagnosis using Structured

Clinical Interview for DSM-IV Axis

1 Disorders (SCID-I) Module PTSD

(First et al., 1995)

Diagnosis using the Composite International Diagnostic

Interview (CIDI) (World Health

Organisation, 1997) and DSM-IV

(APA, 1994) criteria

\section{Rwanda}

Diagnosis using the Composite International Diagnostic

Interview (CIDI) (World Health

Interview (CIDI) (World Health
Organisation, 1997) and DSM-IV

Organisation, 1997)
(APA, 1994) criteria

Turkey, Diagnosis using the clinician

administered PDS with a

combination of DSM-IV (APA,

1994) algorithm and the cut off score of 17

Diagnosis using Structured

Clinical Interview for DSM-IV Axis

1 Disorders (SCID-I) Module PTSD

Not specified Diagnosis using DSM-IV (APA,

1994) criteria with Clinician

1994) criteria with Clinician
Administered PTSD Scale (CAPS)

(Blake et al., 1997)
(HTQ) (Mollica et al., 1992)

Posttraumatic Stress Diagnostic Scale (PDS) (Foa, 1995)

Posttraumatic Stress Diagnostic Scale (PDS) (Foa, 1995)

Posttraumatic Stress Diagnostic Scale (PDS) (Foa, 1995)

Clinician-Administered PTSD

Clinician-Administered PTSD Scale-IV (CAPS) (Blake et al., (PSS-SR) (Foa et al., 1993), Impact of Event Scale-Revised (IES-R) (Weiss \& Marmar, 1997)
Harvard Trauma Questionnair

Scale (CAPS) (Blake et al., 1990) 1997), PTSD Symptomm Scale
Anxiety Sensitivity Index (ASI) (Taylor et al., 1992), Neck Panic Attack Severity Scale (NPASS) and Orthostatic Panic Attack Severity Scale (O-PASS), Neck-Panic Flashback Severity Scale (N-FSS) and Orthostatic-Panic Flashback R Scales (SCL) (Derogatis, 1994)

Hopkins Symptom Checklist-25 (HSCL-25) (Mollica et al., 1990), Anxiety Sensitivity Index (ASI) Vietnamese version (Reiss \& McNally, 1985), Headache Panic Attack Severity Scale (HPASS), Orthostatic Panic Attack Severity Scale (OPASS)

The Demography of Forced Migration

Questionnaire (DFMQ), the Self-Reporting Questionnaire 20 (SRQ-20) (Harding et al., 1980), the psychological health scale from the Medical Outcome Study Self-Report Form (SF-

12) (Ware et al., 1996)

A physical health checklist

Vivo-Checklist of Organised Violence (VCOV), Composite International Diagnostic Interview (CIDI-C) (World Health Organisation, 1997)

Hopkins Symptom Checklist-25 (HSCL-25)

(Derogatis et al., 1974)

Hopkins Symptom Checklist-25 (HSC-25)

(Mollica et al., 1987), Symptom Checklist-90-R (SCL-90-R), Anxiety Sensitivity Index (ASI)

(Taylor et al., 1992)

Anxiety Disorders Interview Schedule-IV (ADISIV) (Brown et al., 1994) including the Hamilton Anxiety Scale (HAS, 1959) and the Hamilton Depression Scale (HDS, 1959). Beck Anxiety Inventory (BAI) (Beck et al., 1988), State Trait Anxiety Inventory (STAI-S+T) (Speilberger et al 1970) Beck Depression Inventory (BDI) (Beck et al., 1961, 1988), World Assumptions (Beck et al., 1961, 1988), World Assumption Quality of Life Inventory (QOLI) (Frisch, 1992) 
Stenmark, Catani,

Neuner, Elbert \& Holen

(2013) Norway

ter Heide, Mooren, Kleijn,

de Jongh \& Kleber (2011)

The Netherlands

ter Heide, Mooren, van

de Schoot, de Jong \&

Kleber (2016) The

Netherlands

\section{Abbreviations:}

EMDR, Stabilisation

$\mathrm{N}=81,56$

males, 25

females, Mean

age $=35$

$\mathrm{N}=20,12$

males, 8

females, Mean

age $=41$

Not

specified

Afghanistan,

Afghanistan

Algeria,

Angola,

Bosnia,
Iraq,

Iraq,

Turkey

$\mathrm{N}=72,52$ male, Not specifie

20 female, Mean

age $=41$
Diagnosis (DSM-IV) using the

Clinician Administered PTSD

Scale (CAPS) (Blake et al., 1995)

Diagnosis using Structured

Clinical Interview for DSM-IV Axis

1 Disorders (SCID-I) Module

PTSD.

Diagnosis using DSM-IV-R (APA,

2000) criteria with Clinician

Administered PTSD Scale (CAPS)

(Blake et al., 1995)
Clinician Administered PTSD

Scale (CAPS) (Blake et al., 1995)

Structured Clinical Interview for

DSM-IV Axis 1 Disorders (SCID-I)

Module PTSD (Dutch version by

Van Groenestijn et al., 1998),

Harvard Trauma Questionnaire

(HTQ) (Mollica et al., 1996a)

Clinician-Administered PTSD

Scale (CAPS) (Blake et al., 1995),

Harvard Trauma Questionnaire

(HTQ) (Mollica et al., 1996a)
M.I.N.I International Neuropsychiatric

Interview (Sheehan et al., 1998), the Hamilton

Rating Scale for Depression (HAM-D)

(Hamilton, 1960)

Mini International Neuropsychiatric Interview

(MINI) (Sheehan et al., 1998) (Dutch version

by Overbeek et al., 1999), Hopkins Symptom

Checklist (HSCL-25) (Mollica et al, 1996b),

World Health Organisation Quality of Life

questionnaire (WHOQOL-BREF) (WHOQOL

Group, 1998)

Hopkins Symptom Checklist-25 (HSCL-25)

(Derogatis et al., 1974), World Health

Organisation Quality of Life Assessment

(WHOQOL-BREF) (WHO, 1998) with antidepressant medications.

a Study used a 2 × 2 design. Participants received experimental and control psychological interventions in combination with antidepressant medications. 
Table 2. Study characteristics related to SURE and Cochrane's risk of bias criteria. (Cochrane criteria within border)

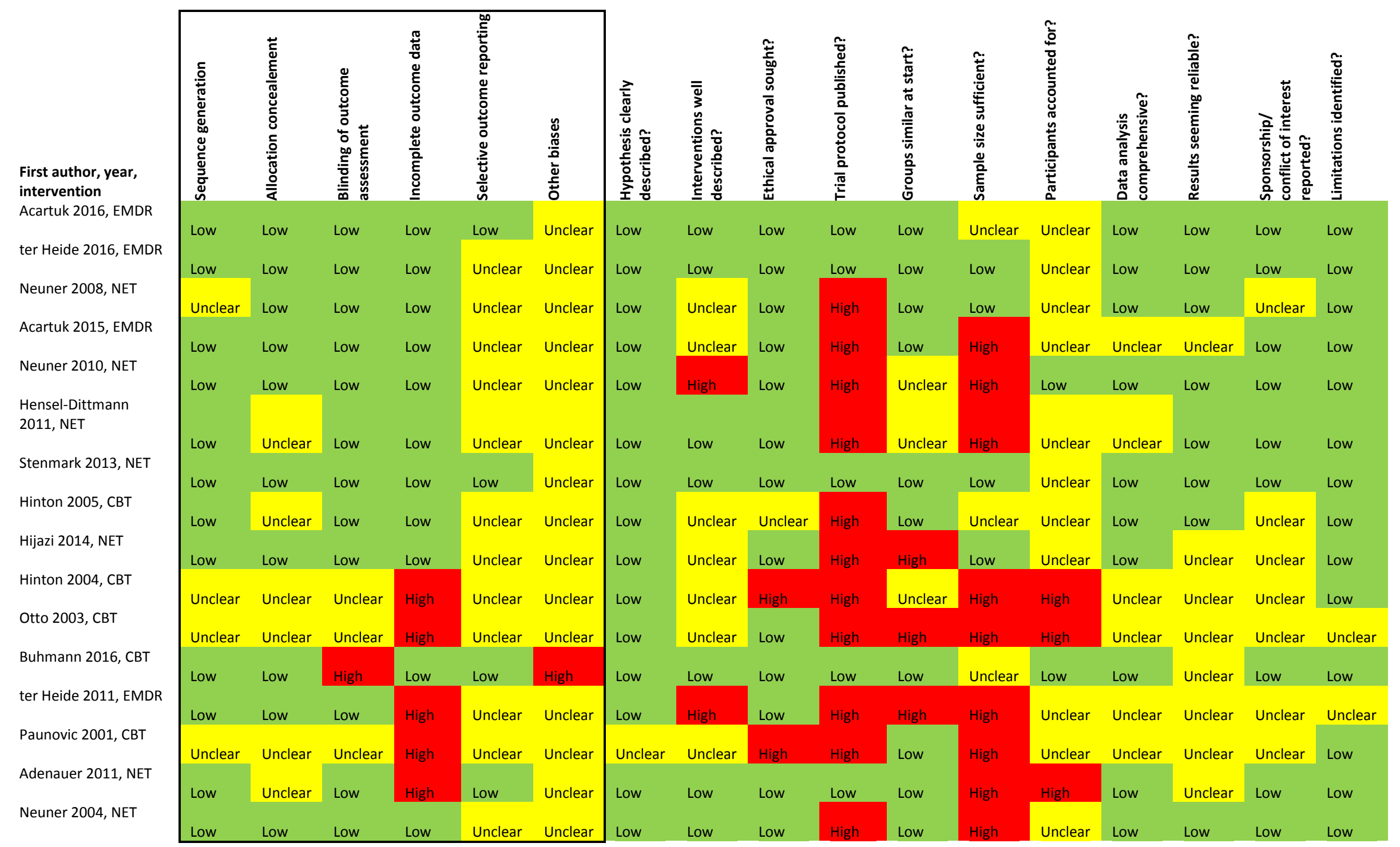


Table 3. Efficacy of experimental intervention versus inactive and active controls for PTSD severity with GRADE judgments of evidence quality.

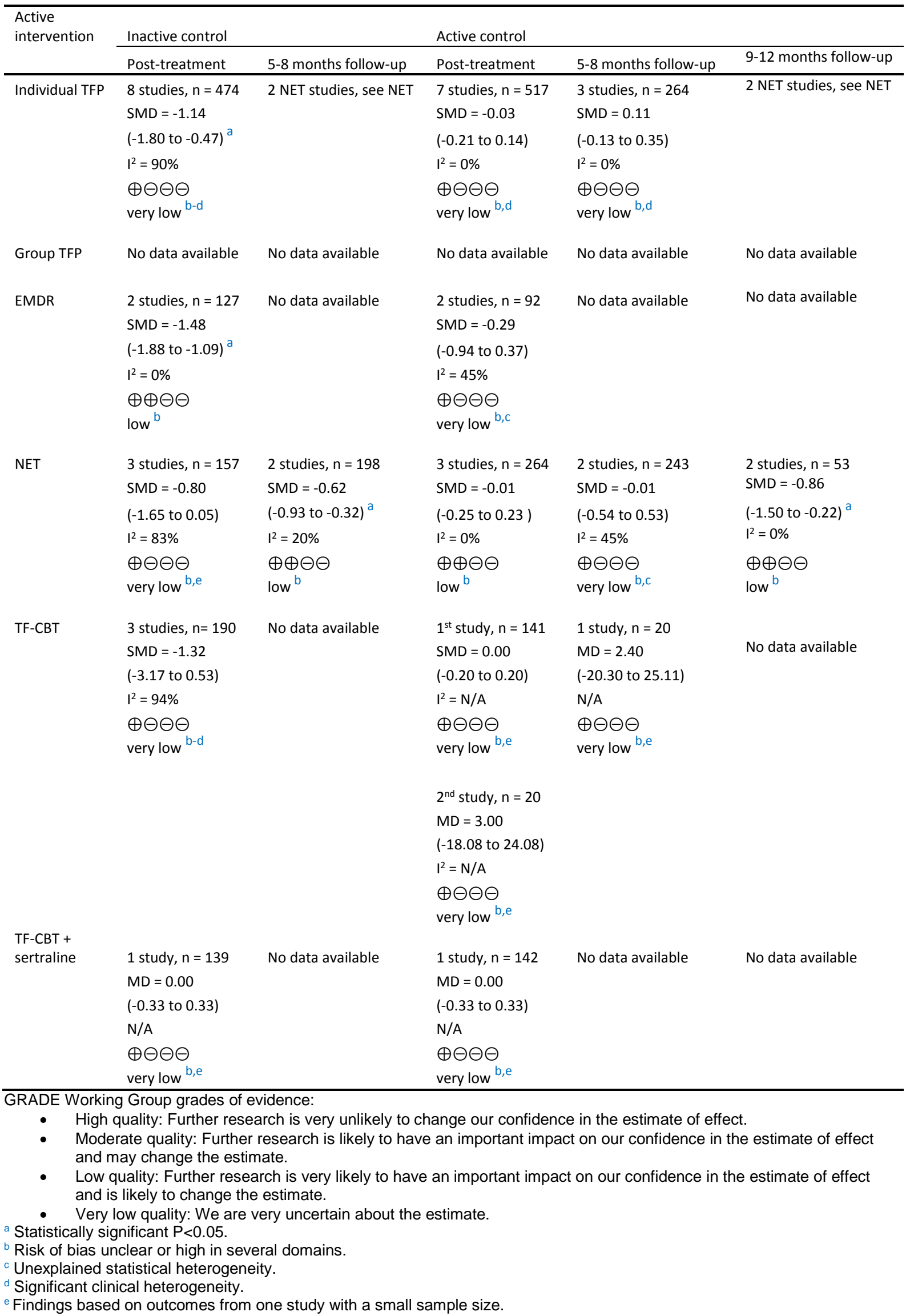




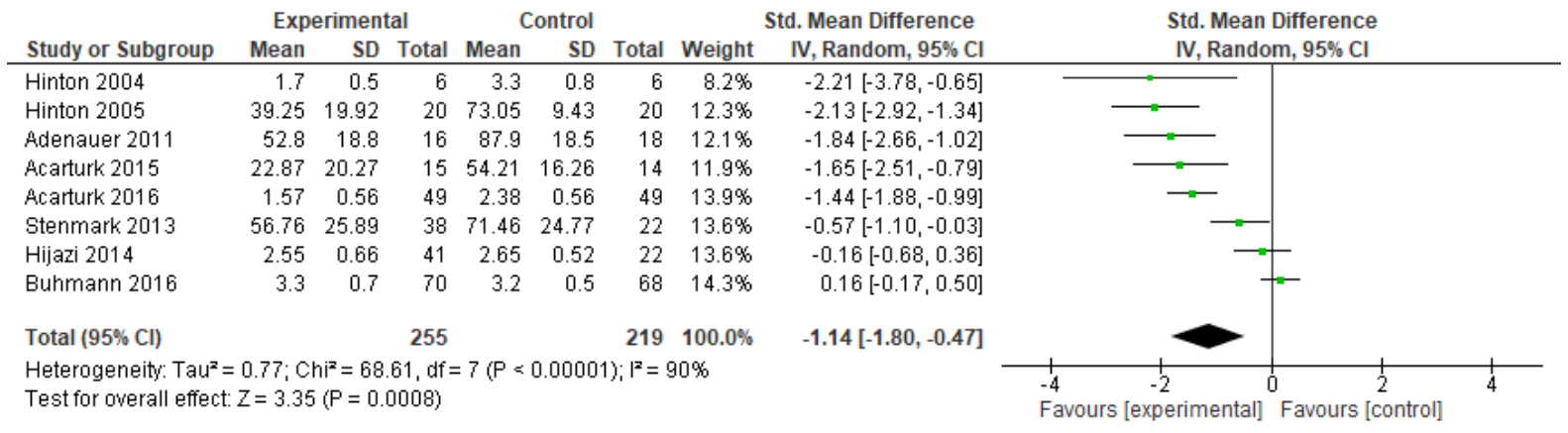

Figure 2. Forest plot of comparison: trauma-focused psychotherapy vs inactive control, main outcome: PTSD severity at 0 to 4 months

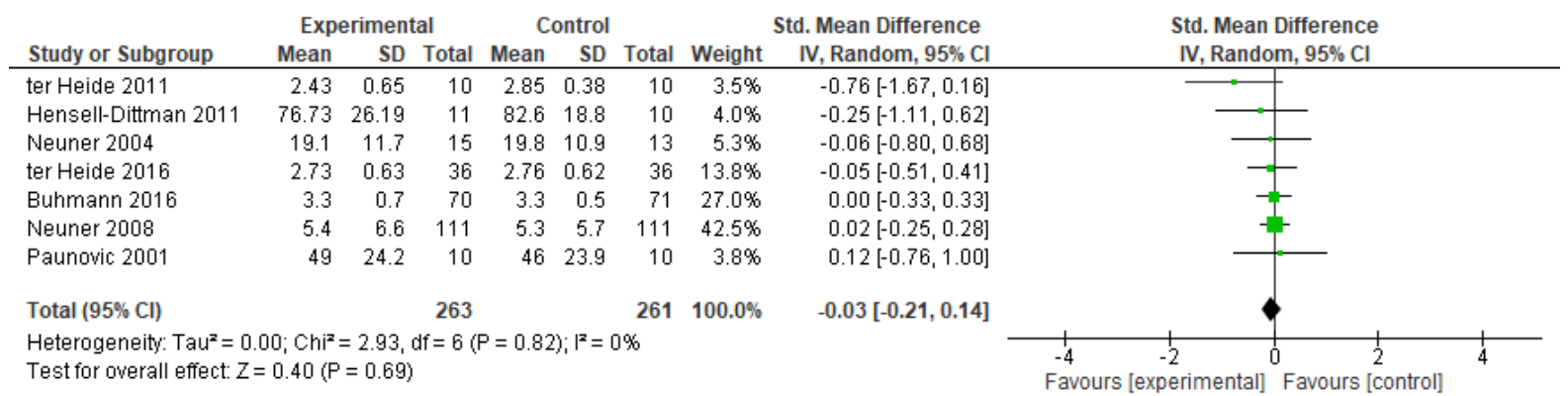

Figure 3. Forest plot of comparison: trauma-focused psychotherapy vs active control, main outcome: PTSD symptoms at 0 to 4 months 